\title{
Synthesis of a dinuclear palladacycle with a doubly metallated ligand and its reactivity towards diphosphines ${ }^{\dagger}$
}

\author{
Pablo Romarís ${ }^{1, *}$, Fátima Lucio-Martínez ${ }^{1}$, Francisco Reigosa ${ }^{1}$, Paula Munín ${ }^{1}$, Paula Polo-Ces ${ }^{1}$, \\ María Teresa Pereira ${ }^{1}$ and Jose Manuel Vila ${ }^{1, *}$ \\ 1 Department of Inorganic Chemistry, Faculty of Chemistry, University of Santiago de Compostela, Avd. das \\ Ciencias, s/n, 15782 Santiago de Compostela, Spain; e-mail@e-mail.com \\ * pablo.romaris@rai.usc.es; Tel.: +34-881-814-442 \\ * josemanuel.vila@usc.es; Tel.: +34-881-814-442 \\ + The $22^{\text {nd }}$ International Electronic Conference on Synthetic Organic Chemistry, 15 November- 15 December \\ 2018.
}

Received: date; Accepted: date; Published: date

\begin{abstract}
Palladacycles are an important class of organometallic compounds. They are interesting for their stability and for their important applications as catalysts in cross-coupling reactions. The work described herein relates to the synthesis of a dicyclometallated compound stemming from a diamine ligand, with a doubly metallated aromatic ring. Its reactivity towards a variety of tertiary diphosphines is also considered. The latter ligands show diverse coordination modes, opening the possibility of synthesizing quite different complex structures bearing transition metal atoms in close proximity to each other.
\end{abstract}

Keywords: palladium; cyclometallation; diimine; polymetallic complexes

\section{Introduction}

In the past decades cyclometallated transition metal complexes have attracted much attention. These cyclometallated compounds usually form five-membered rings [1,2] with a great variety of transition and non-transition metals, although palladacycles are the most common due to its stability and ease of synthesis, apart from their useful applications. Most ligands that can be cyclometallated contain $\mathrm{N}$ atoms although many other different ligands have been cyclometallated successfully

Cyclometallated compounds have applications based on their mesogenic[3], luminescent and electronic[4,5] properties, and they also have potential applications in medicine[6]. The most important application of these compounds, especially palladacycles, is as catalysts in a variety of synthetic processes. Palladacycles present interesting applications for synthetic organic chemistry as efficient catalysts in a variety of carbon-carbon cross-coupling reactions[7,8], which have led to an increase in interest on these compounds in the last years. For catalytic applications, often control and modulation of their activity by other ligands is required. Tertiary phosphines have been the most frequently used ligands for this role.

We have researched extensively terdentate $[\mathrm{C}, \mathrm{N}, \mathrm{X}](\mathrm{X}=\mathrm{O}, \mathrm{N})$ ligands, but also bidentate $[\mathrm{C}, \mathrm{N}]$ ligands, like imines[9]. In this line of research there have been studies of doubly cyclometallated compounds [10,11]. These compounds can react with polydentate ligands such as phosphinesobtaining interesting polymetallic structures, formed by cyclometallated units joined by bidentate ligands [12]. This work belongs to this line of research, investigating the reactivity of a doubly metallated palladacycle with diphosphines, which can then lead to the synthesis of new complex structures. 


\section{Materials and Methods}

The diimine ligand was prepared by condensation of a dialdehyde with two amine molecules. 2-(methylthio)aniline was added to a solution of terephtaldehyde in chloroform. The reaction was heated $8 \mathrm{~h}$ under reflux in amodified Dean-Stark setup. The solvent was removed under vacuum, obtaining a yellow solid.<smiles>CSc1ccccc1/N=C/c1ccc(/C=N/c2ccccc2SC)cc1</smiles>

Figure 1.

The cyclometallated compound was prepared by the dropwise addition of a solution of palladium acetate in toluene to a solution of the ligand in toluene, under nitrogen atmosphere. The reaction was then heated for $24 \mathrm{~h}$ to $60^{\circ} \mathrm{C}$. The reaction mixture is centrifuged, obtaining a red solid.

In order to purify the compound, a continuous solid-liquid extraction with dichlorometanewas performed using a Soxhlet extractor. This liquid phase was dried, obtaining a red solid which is the cyclometallated compound.<smiles>CSc1ccccc1/N=C/c1ccc(/C=N/c2ccccc2SC)cc1</smiles>

Figure 2.

The cyclometallated compound, with an acetate ligand coordinated to the palladium, was reacted with $\mathrm{NaCl}$ in a ligand exchange reaction, to obtain the compound with chloro ligands, which can be more conveniently removed for the reactions with phosphines. The compound dissolved in acetone was mixed with a $\mathrm{NaCl}$ solution, and the reaction was stirred at room temperature for $24 \mathrm{~h}$ and centrifuged.The red solid obtained is washed with water to eliminate any excess $\mathrm{NaCl}$ and dried.

The synthesis of phosphine derivatives has followed a general method: the cyclometallated compound has been reacted in acetone solution, with an excess of silver perchlorate to remove the chlorine ligand and phosphine in the appropriate phosphine:palladacycle stoichiometric ratio. The reaction mixture is then centrifuged and the solvent is evaporated, obtaining the products which do react.

Before using different diphosphines a reaction with deuterated tryphenylphoshine has been carried out to confirm that the compound has not decomposed or degraded in the subsequent reaction. Due to the poor solubility of the palladacycle with chloro ligands its structure could not be confirmed unambiguously by NMR

Table 1.Reaction conditions for the reactions with phosphines

\begin{tabular}{ccccc}
\hline Product & Phosphine & Ratio & AgClO $_{4}$ & Temperature $\left(^{\circ} \mathbf{C}\right)$ \\
\hline 5 & dppm & $2: 1$ & $2: 1$ & r. t. \\
6 & dppm & $2: 1$ & - & r. t. \\
7 & vdpp & $1: 1$ & $2: 1$ & 50 \\
8 & dppf & $2: 1$ & $2: 1$ & r. t. \\
\hline
\end{tabular}

Three factors have been studied in these reactions. The first and most important was the stoichiometric ratio of phosphine used. It is expected that the reactions with phosphine in a 2:1 ratio to the metallacycle should lead to compounds with the diphosphine acting as a chelate or 
monodentate ligand, while reactions with a 1:1 ratio of phoshine to metallacycle should lead to compounds with diphosphines as bridging ligands.

The other factors studied were the temperature and the presence or not of a precipitating agent. Three different diphosphines have been used and, despite some exceptions, most successful reactions have occurred at room temperature and with a precipitating agent. This seems to confirm that the precipitating agent is important in the replacement of the chloro ligand, and that a higher temperature is detrimental to the reaction.

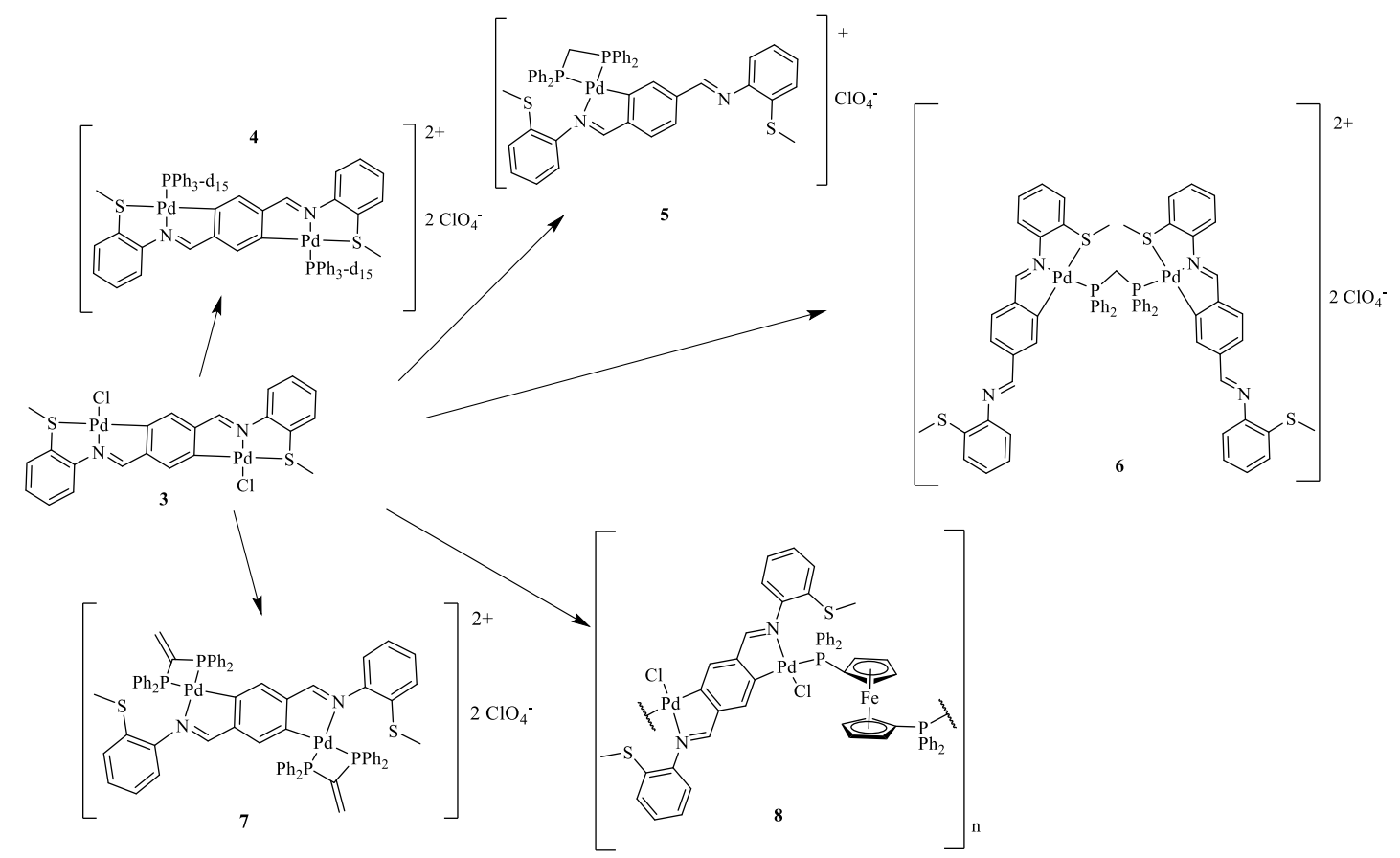

Figure 3. 


\section{Results and Discussion}

The techniques of NMR spectroscopy and IR spectroscopy have been used to characterize the compounds. The NMR spectra have been done in DMSO, as the compounds were not soluble on $\mathrm{CDCl}_{3}$ or decomposed on it, like the ligand.

The comparison of the ${ }^{1} \mathrm{H}$ NMR spectra of the ligand and its cyclometallated compound made clear the double metalation of the aromatic ring. A general deshielding effect is seen on the cyclometallated compound due to the electron donation to the metal, especially in the shift of the signals corresponding to the imine proton and to the thyomethyl group. As the protonscorresponding to the methylthioaniline ring and the central ring can be easily differentiated, by looking at the integrals it can be seen that two protons on the central aromatic ring have disappeared because of the metalation.

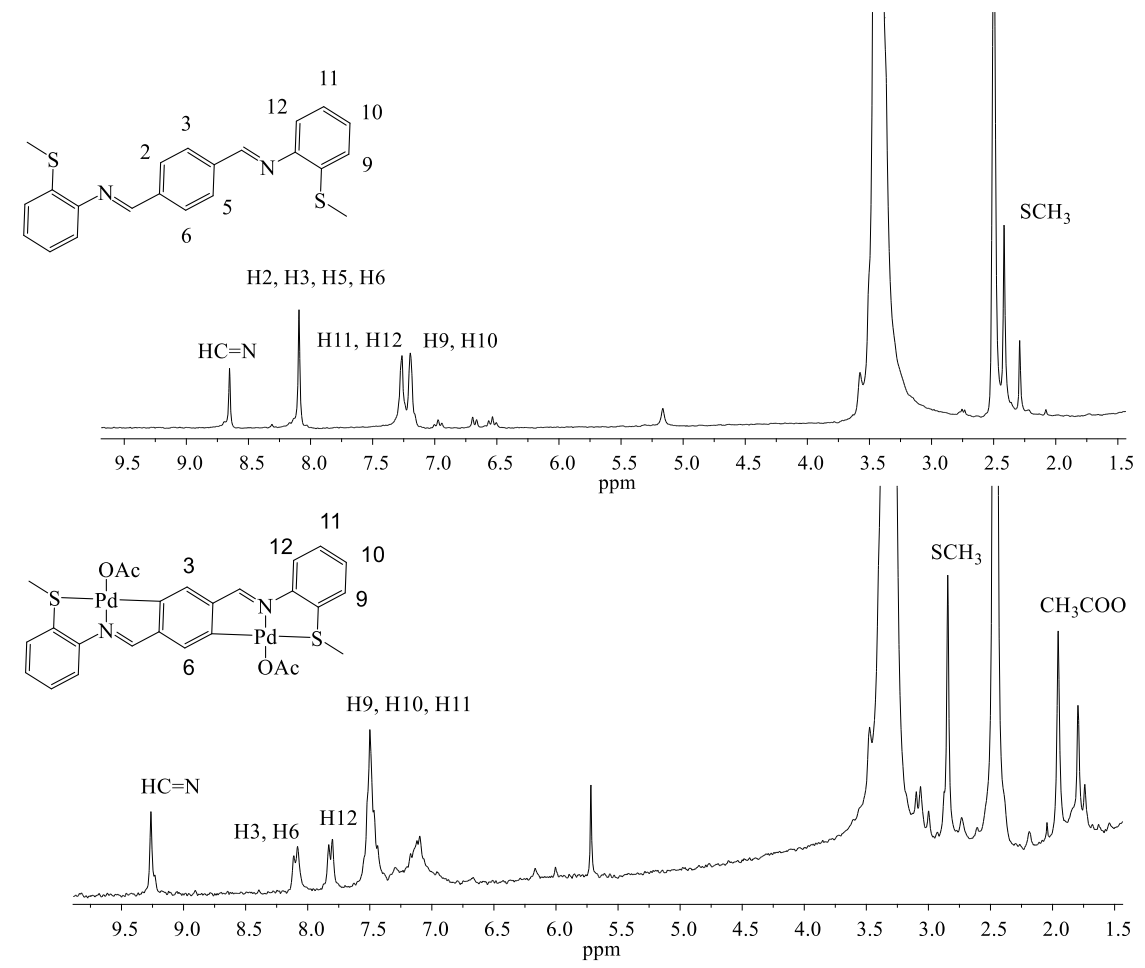

Figure 4 .

The IR spectrum of the ligand shows a band corresponding to the $\mathrm{C}=\mathrm{N}$ bond. The same band appears in the IR spectrum of the cyclometallated compound, although slightly shifted to smaller wavenumbers. This confirms the metalation and shows that the ligand coordinates the metal through the nitrogen lone pair; if coordination was through the $\pi$ electrons of the double bond, there would be a larger shift in the wavenumber. In the spectrum, bands corresponding to the acetate ligand also show up.

The ${ }^{1} \mathrm{H}$ NMR spectrum of compound 3 is very similar to the acetate cyclometallated compound, although the poor solubility makes the compound difficult to characterise. The only difference is the disappearance of a signal corresponding to the acetate ligand.

The compounds with phosphines were characterized with ${ }^{31} \mathrm{P} N M R$, apart from ${ }^{1} \mathrm{H} N M R$ and IR spectroscopy. It allowsto obtain information about the compounds even when the proton NMR is very difficult to interpret. 
The ${ }^{1} \mathrm{H}$ spectrum shows the iminic proton as a doublet due to ${ }^{4} \mathrm{~J}$ coupling with the phosphorus atoms. The peak for the H3- H6 protons is also shifted upfield due to the electronic effects produced by the aromatic rings of the phosphine nearby, and is also a doublet. The other signals are similar. In the ${ }^{31} \mathrm{P}$ NMR spectrum a single peak at positive chemical shift which indicates coordination to a metal is observed.

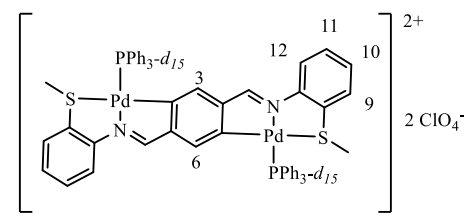

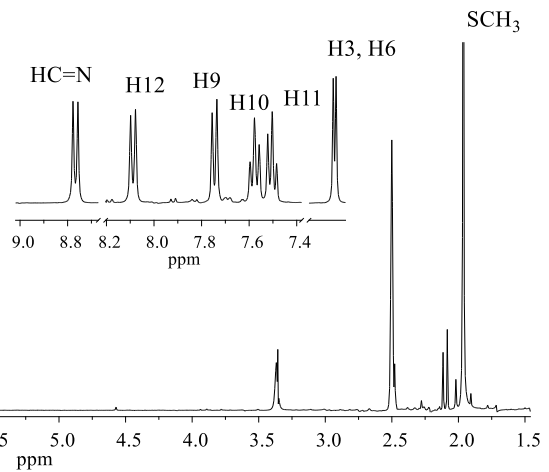

Figure 5.

In the ${ }^{31} \mathrm{P}$ NMR spectrum of compound 4, two peaks at positive shifts appear. These peaks are doublets with the same, small coupling constant of $5 \mathrm{~Hz}$. This indicates that both phosphorus atoms are not equivalent but they are coupled to each other and both coordinate to a metal atom, meaning that the dppm is acting as a chelate ligand. An additional peak, a singlet at negative shift appears, which corresponds to the complex $\operatorname{Pd}(\mathrm{dppm}) \mathrm{Cl}_{2}$.

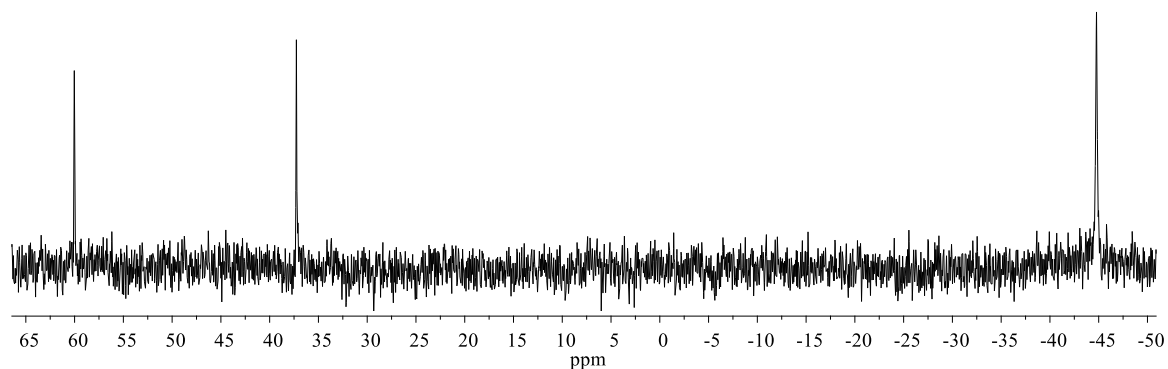

Figure 6.

The proton NMR also shows clues about this structure. Two peaks in the high field region corresponding to the two $\mathrm{SCH}_{3}$ groups are observed, one with the same shift as in the cyclometallated compound, the other similar to the ligand $\mathrm{SCH}_{3}$ group, not coordinated to a metal. In the low field region of the spectrum it is difficult to see the peaks due to the aromatic protons of the phosphine phenyl groups, but the peak corresponding to the iminic proton coordinated to the metal is observed.

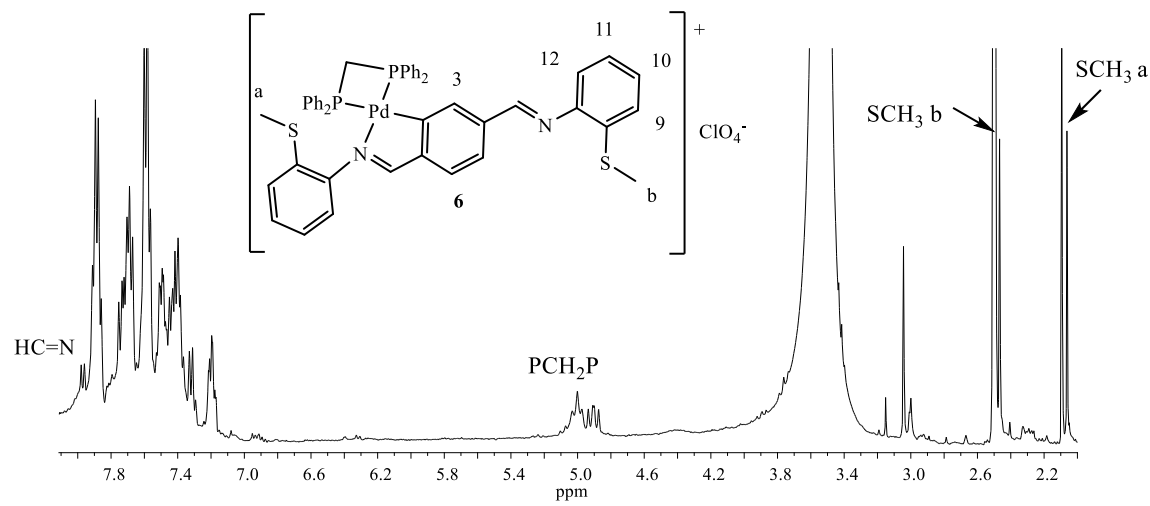

Figure 7. 
When the reaction conditions are changed, without using the precipitating agent, the results change significantly. In the ${ }^{31} \mathrm{P}$ NMR a single peak is observed at positive shift which indicates coordination to the metal. And as before there is another peak at a very low shift which corresponds to the compound $\mathrm{Pd}(\mathrm{dppm}) \mathrm{Cl}_{2}$. This result, where the $\mathrm{P}$ atoms are equivalent, can be explained by a structure in which the diphosphine acts as a bridging ligand between two cyclometallated molecules, thus being equivalent.

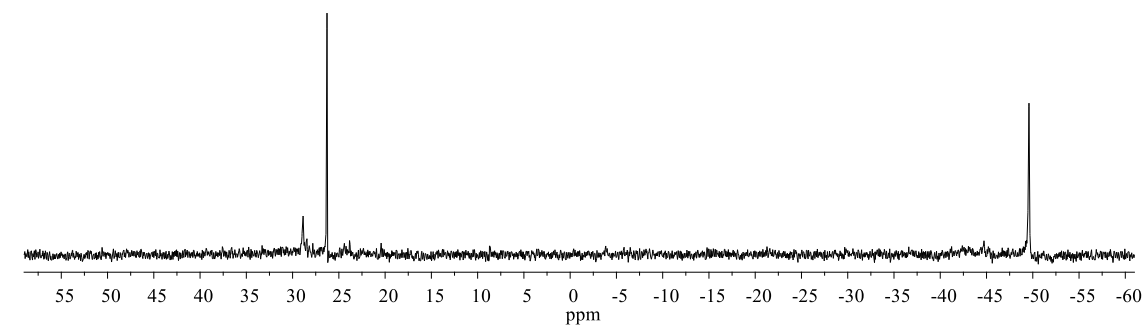

Figure 8.

In the successful reaction with vdpp, unlike the other reactions the phosphine was used in a 1:1 ratio. The product seems to be a chelate as it would be expected. This is clearly reflected on the ${ }^{31} \mathrm{P}$ NMR, where there are two doublets at positive chemical shift.

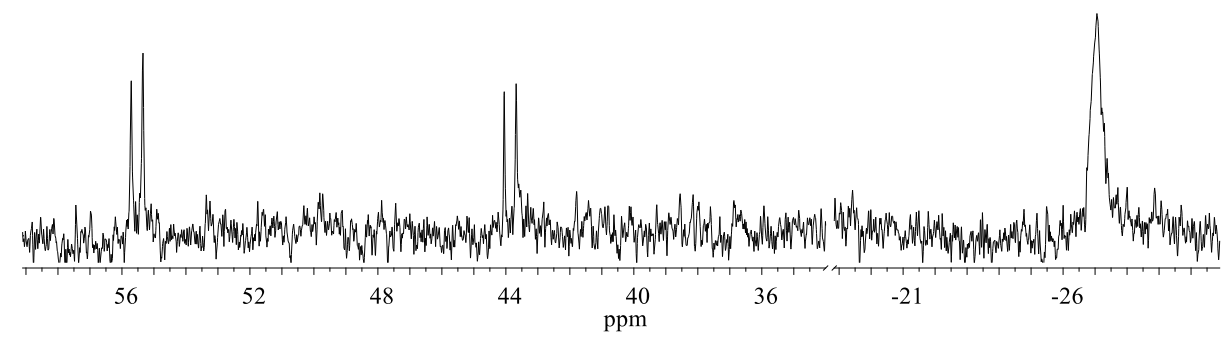

Figure 9.

In the proton NMR unlike the previous NMR, only one signal corresponding to the $\mathrm{SCH}_{3}$ groups is observed. Its chemical shift is in agreement with a $\mathrm{SCH}_{3}$ group not coordinated to the metal. The low field region of the spectrum it is difficult to interpret due to all the aromatic protons of the coordinated phosphine appearing, but one can see at 6,5-7,0 ppm the two protons of the coordinated phosphine appearing as a complex multiplet (the two alkene protons are not magnetically equivalent)

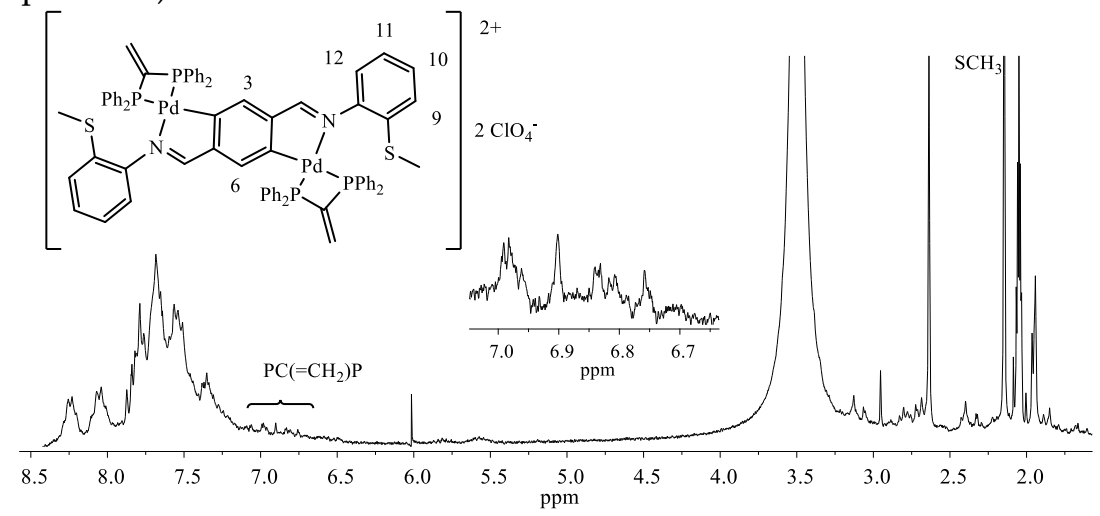

Figure 10. 
The ${ }^{31} \mathrm{P}$ NMR of compound 8 shows one peak at a positive shift, indicating that the phosphine acts as a bridging ligand. However unlike the compound with dppm 4 only one peak corresponding to the $\mathrm{SCH}_{3}$ group is observed. Its shift corresponds to a sulfur atom coordinated to the metal. This means the compound has a polymeric structure with the cyclometallated units linked to one another by the diphosphine. This structure may seem uncommon but polymeric structures like this have been reported for metallacycles.

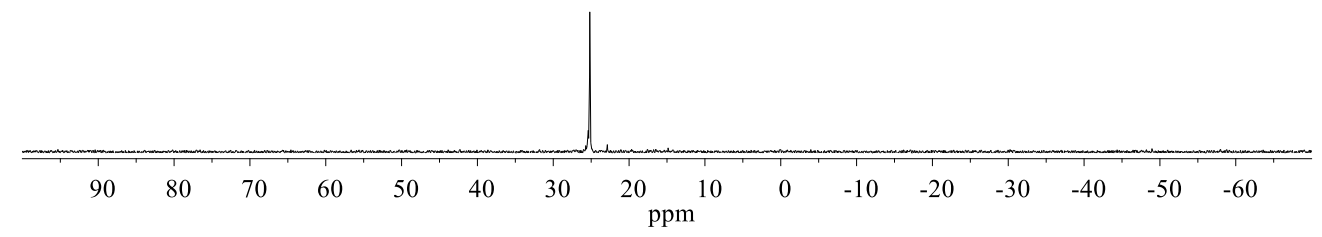

Figure 11.

In the ${ }^{1} \mathrm{H}$ NMR spectrum the two singlets corresponding to the cyclopentadienyl rings are clearly observed. The peaks are deshielded compared to the free phosphine, as it is expected in a compound where the diphosphine is coordinated to a metal. The chemical shift of the peak corresponding to the $\mathrm{SCH}_{3}$ group also indicates that it is not coordinated to a metal

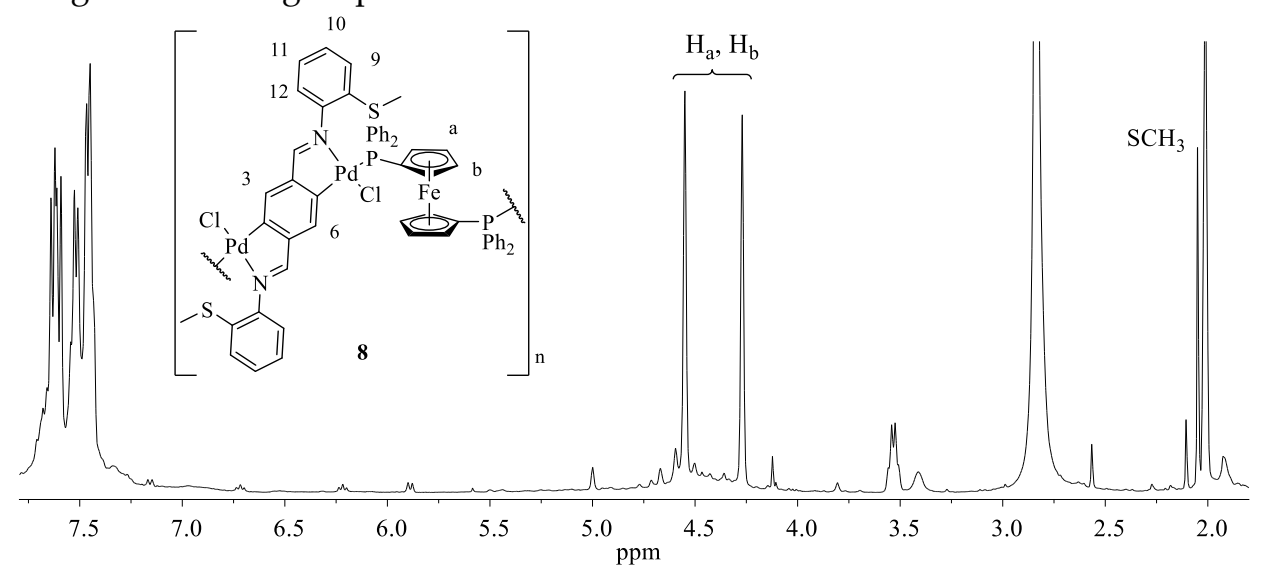

Figure 12.

\section{Conclusions}

The synthesis of a doubly metallated palladacycle was accomplished. The reaction of this metallacycle with diphosphines yields compounds where theseligands exhibit different coordination modes, depending on the characteristics of each phosphine and the reaction conditions.

Acknowledgments: We wish to thank the financial support received from the Xunta de Galicia (Galicia, Spain) under the Grupos de Referencia Competitiva Programme Projects GRC2015/009. F. Reigosa and F. Lucio thank the Spanish Ministry of Education (grant FPU15/07145 and FPU13/05014)

Conflicts of Interest: The authors declare no conflict of interest. The founding sponsors had no role in the design of the study; in the collection, analyses, or interpretation of data; in the writing of the manuscript, and in the decision to publish the results. 


\section{References}

1. Omae I. Intramolecular five-membered ring compounds and their applications. Coord. Chem. Rev.2004248, 995-1023

2. Dupont J.; Pfeffer M. (Eds).Palladacycles, WILEY-VCH, London, 2008.

3. Wang Y.; Liu Y.; Luo J.; Qi H.; Li X.; Nin M.; Liu M.; Shi D.; Zhu W.; Cao Y. Metallomesogens based on platinum(II) complexes: synthesis, luminescence and polarized emission. Dalton Trans. 201140, 5046-5051.

4. HisslerM.; McGarrahJ. E.; ConnickW. B.; GeigerD. K.; CummingsS. D.; EisenbergR. Platinum diimine complexes: towards a molecular photochemical device. Coord. Chem. Rev.2000208, 115-137 10.1016/S0010-8545(00)00254-X

5. XingY.; LiuC.; XiuJ.; Li,J. Photostable fluorophenyl-substituted cyclometallated platinum(II) emitters for monitoring of molecular oxygen in real time.Inorg. Chem.2015, 54, 7783-7790 10.1021/acs.inorgchem.5b00775

6. Gigli R.; Pereira G.J.S.; Antunes F.; Bechara A.; Garcia D. M.; Spindola D. G.; Jasiulionis M. G.; Caires A.C.F.; Smaili S. S.; BincolettoC. The biphosphinic paladacycle complex induces melanoma cell death through lysosomal-mitochondrial axis modulation and impaired autophagy. Eur.J. Med. Chem.2016107, 245-254.10.1016/j.ejmech.2015.11.008

7. Herrmann W. A.; Brossmer C.; Ofele K.; Beller M.; Fischer H. Coordination chemistry and mechanism of metal-catalyzed C-C coupling reactions. Part 6. Palladacycles as efficient catalysts for aryl coupling reactions. Angew. Chem. Int. Ed. 199534, 1848-9. 10.1002/anie.199518481

8. NavarroO.; MarionN.; OonishiY.; Kelly IIIR.A.; NolanS.P.Suzuki-Miyaura, $\alpha$-Ketone Arylation and Dehalogenation Reactions Catalyzed by a Versatile N-Heterocyclic Carbene-Palladacycle Complex.J. Org. Chem. 200671, 685-692

9. Naya L.; Vazquez-Garcia D.; Fernández A.; López-Torres M.; Marcos I.; Lenis O.A.; Pereira M.T.; Vila J.M. Novel palladacycle N-heterocyclic carbene complexes with bidentate [C,N] and terdentate [C,N,N] and $[\mathrm{C}, \mathrm{N}, \mathrm{O}]$ Schiff bases. Synthesis, characterization and crystal structure analysis. J. Organomet. Chem.2014772, 192-201 10.1016/j.jorganchem.2014.07.018

10. Frieiro Gomis, P. Preparación y estudio estructural de compuestos de paladio (II). Aplicaciones en catálisis. PhD Dissertation, University of Santiago de Compostela, 2015

11. Polo Ces, P. Síntesis y caracterización de nuevos compuestos de Pd (II) con ligandos nitrogenados. Aplicaciones en catálisis. PhD Dissertation, University of Santiago de Compostela, 2016

12. Frieiro-Gomis P.; Lucio-Martinez F.; Munin-Cruz P.;Ortigueira J. M.; Pereira M. T.; Polo-Ces P.; Vazquez-Garcia D.; Vila J.M. The chelate-to-bridging shift of phosphane dipalladacycles: convenient synthesis of double A-frame tetranuclear complexes. Chem Commun. 2018, 54, 2662-2665, 10.1039/C8CC01046C

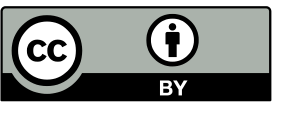

(C) 2018 by the authors. Submitted for possible open access publication under the terms and conditions of the Creative Commons Attribution (CC BY) license (http://creativecommons.org/licenses/by/4.0/) 